\title{
Chromospheric models of solar analogues with different activity levels
}

\begin{abstract}
M. Vieytes, P. Mauas, and C. Cincunegui
Instituto de Astronomía y Física del Espacio, CC. 67 Suc. 28 (1428) Buenos Aires, Argentina

e-mail: mariela@iafe.uba.ar

Received 6 January 2005 / Accepted 24 april 2005

Abstract. We computed chromospheric models of the Sun as a star and of nine solar analogues. The atmospheric models were constructed to obtain the best possible match with the Ca II K and $\mathrm{H} \beta$ lines, including the asymmetry of the lines due to macroscopic velocity fields. The stars were chosen with $0.62<B-V<0.68$ (the solar $B-V=0.65$ ) and have a wide variety of magnetic activity levels, which allows us to study the differences in atmospheric structures induced by activity. For the less active stars we found that the changes with activity are in the region of the temperature minimum, while the most active stars show changes all along their atmospheric structures, mainly in the upper chromosphere. Regarding the macroscopic velocity fields, we can distinguish between the two groups. The most active group has a velocity field in the temperature-minimum region, and the other group in the chromospheric plateau. We also computed the net radiative losses for each model, and found that they depend linearly on the usual index of chromospheric activity, $S_{\text {CaII }}$.
\end{abstract}

Key words. radiative transfer - stars: atmospheres - stars: activity

\section{Introduction}

The solar chromospheric models most used in the last twenty years are the ones by Vernazza et al. (1981), as modified later by Fontenla et al. (1993). The models in these papers represent different brightness components of the solar chromosphere (quiet cell, magnetic network and active region) and in particular model $\mathrm{C}$ represents the average quiet Sun. These are semiempirical models constructed to match as well as possible the observed profiles of a large number of spectral lines and the continuum in a wide range of wavelengths, with the intensities averaged both temporally and spatially.

In this kind of modelling there are no hypotheses made about the physical processes responsible for the phenomena observed in the solar atmosphere. Semi-empirical models can indicate only the general properties of the temperature and mechanical heating distributions yielding a given spectrum. Nevertheless, much useful information was extracted from these models and improved our knowledge about the Sun.

A new set of dynamical models, based on this knowledge, try to reproduce the solar observations from a theoretical point of view. For example the models by Carlsson, Stein and collaborators (e.g., Carlsson \& Stein 1997) are hydrodynamical models that simulate dynamical processes and use their properties to supply the mechanical heating to reproduce the observations. To the extent that the hydrodynamical models agree with observations they tell us more about the physical mechanisms at work than can be learned from the semi-empirical models. These hydrodynamical models are useful to reproduce the basal emission, not the emission from an active star.

Stellar chromospheric modelling, however, is still in its beginnings. For example, Thatcher et al. (1991) built a semiempirical model of the chromosphere of the dK2 star $\varepsilon$ Eridani, matching flux profiles of the $\mathrm{Ca}$ IR triplet lines, $\mathrm{H} \alpha, \mathrm{H} \beta$ and the Na D lines.

A grid of chromospheric models for dM stars of different activity levels has been used by Houdebine \& Doyle (1994a,b) and Houdebine et al. (1995, 1996), to study the response of the hydrogen spectrum to different atmospheric parameters.

Semi-empirical models of AD Leo (dM3.5 star) in its quiescent state and during a large flare were constructed by Mauas \& Falchi (1994) and Mauas \& Falchi (1996) respectively. In the same way, models of the dM3-4 stars G1588 and G1628 were constructed by Mauas et al. (1997). These stars are considered as "basal" stars due to their low chromospheric activity level.

Franchini et al. (1998) and Morossi et al. (2003), using IUE and HST ultraviolet spectra, derived empirical temperature distributions in the atmosphere of $\mathrm{G}$ and K-type stars, paying particular attention to the temperature minimum region. They compared different atmospheric models and obtained the best agreement with observations with a temperature increasing outward of the temperature minimum zone.

Vieytes \& Mauas (2004) built a grid of semi-empirical models of G-type stars to study the Vaughan-Preston Gap. They found that the flux in the $\mathrm{Ca}$ II $\mathrm{H}$ and $\mathrm{K}$ lines changes 
nonlinearly when the temperature in the chromosphere is increased, a fact that could explain the existence of the Gap.

There is, however, little information about how the solar atmosphere is modified when the activity level increases. As a first step in this direction, in this work we build semi-empirical models for stars of different activity levels, and we compare them with the one for the Sun as a star.

This paper is arranged as follows: the observational data are discussed in Sect. 2. In Sect. 3 we explain how the modelling was done, we present the computed models and we compare the predicted and observed spectral features. In Sect. 4 we compute the energy requirements of the models. Finally, in Sect. 5 we discuss the results.

\section{The stellar sample}

The Sun is a star of spectral type G2 V, with a $T_{\text {eff }}=5777 \mathrm{~K}$. Neckel (1986) derived the solar $(B-V)_{\odot}=0.650 \pm 0.005,(U-$ $V)_{\odot}=0.195 \pm 0.005$ and $M_{V \odot}=4.82 \pm 0.025$. Porto de Mello $\&$ da Silva (1997) obtained with a different method $(B-V)_{\odot}=$ $0.648 \pm 0.006$ and $(U-V)_{\odot}=0.178 \pm 0.013$. The rotation period is 25 days.

On the Sun and solar-type stars the emission cores of the $\mathrm{Ca}$ II $\mathrm{H}$ and $\mathrm{K}$ doublet, in the visible violet, increase with increasing surface magnetism, i.e. with increasing chromospheric activity. The most widely used activity indicator is the $S_{\text {CaII }}$ index, which is the ratio of the fluxes in the $\mathrm{H}$ and $\mathrm{K}$ passbands, and two nearby reference passbands $20 \AA$ wide (Baliunas et al. 1998). The mean $S_{\text {CaII }}$ for the Sun over the last three solar cycles is 0.179 , ranging from 0.160 to 0.205 in its normal state of activity (Baliunas et al. 1995). These values imply that the Sun is a star with a low level of chromospheric activity.

The stars we modelled were chosen to have similar colours to the Sun and therefore similar photospheric structures, but different chromospheric activity levels. This fact implies different ages and/or rotation periods. These stars can be considered as solar analogues, since they share some characteristics with the Sun. Table 1 shows some of the stellar parameters. In particular, the two rightmost columns of Table 1 show the mean values of the $S_{\mathrm{MW}}$ index obtained at Cerro Tololo InterAmerican Observatory (Henry et al. 1996), and from our spectra (Cincunegui \& Mauas 2004).

Henry et al. (1996) used the Cassegrain Spectrograph on the $1.5 \mathrm{~m}$ telescope at CTIO. Due to the low instrumental resolution $(\lambda / \Delta \lambda \approx 2500)$ the HK-passbands were chosen $3.28 \AA$ wide (4 pixels). To calibrate their measurements with the Mount Wilson ones, 22 stars with more than $100 \mathrm{ob}-$ servations at MW and with activity levels almost constant were used as calibration stars.

We observed 18 of these calibration stars in several observing runs (typically between 8 and 12 observations for each star), and measured the weighted fluxes in two passbands centered in the $\mathrm{H}$ and $\mathrm{K}$ lines, using a weight function that mimics the instrumental profile at Mount Wilson. We also measured the average flux in two continuum passbands nearby, and calculate an $S^{\prime}$ index as the ratio between the weighted
Table 1. Stellar characteristics (from Cincunegui \& Mauas 2004). The last two columns give the $S_{\text {CaII }}$ measured by Henry et al. (1996) at CTIO and by Cincunegui \& Mauas (2004) at CASLEO.

\begin{tabular}{l|cccccc}
\hline \hline HD & $S$. type & $B-V$ & $U-B$ & {$[\mathrm{Fe} / \mathrm{H}]$} & $S_{\text {Сті }}$ & $S_{\mathrm{CM}}$ \\
\hline Sun & G2 V & 0.65 & 0.195 & 0 & 0.179 & - \\
189567 & G3 V & 0.64 & 0.07 & -0.30 & 0.238 & 0.17 \\
197076 & G5 V & 0.63 & 0.09 & - & - & 0.19 \\
172051 & G5 V & 0.63 & 0.17 & - & 0.181 & 0.19 \\
146233 & G2 Va & 0.65 & 0.16 & 0.05 & - & 0.19 \\
19467 & G3 V & 0.65 & 0.15 & - & - & 0.16 \\
20766 & G2.5 V & 0.64 & 0.08 & -0.22 & 0.245 & 0.25 \\
30495 & G3 V & 0.64 & 0.13 & -0.13 & 0.286 & 0.34 \\
1835 & G3 V & 0.67 & 0.22 & -0.01 & 0.344 & 0.35 \\
202917 & G5 V & 0.65 & - & - & 0.743 & 0.76 \\
\hline
\end{tabular}

fluxes in the line centers and the average fluxes in the continua. For each calibration star, we average its $S^{\prime}$ values. Finally, we fit our indexes with the Mount Wilson ones, finding that $S_{\mathrm{MW}}=1.277 S^{\prime}-0.002$.

In the rest of this section we describe the stars in our sample. HD 189567 is a star of spectral type G3 V with $B-V=$ 0.64. This star was part of a survey of Ca II chromospheric emission in southern solar-type stars made by Henry et al. (1996), and has low chromospheric activity.

HD 197076 is a star of spectral type G5 V with $B-V=$ 0.63. The rotation period is 19 days (Wright et al. 2004), which means that it rotates faster than the Sun. However, it has an $S_{\text {CaII }}$ similar to the solar one. This star was observed at the Mount Wilson Observatory at least on four occasions (Duncan et al. 1991), and was selected by the SETI program as a target to transmit the TAM message.

Another low chromospheric activity star is HD 172051. This is a G5 V type star with $B-V=0.63$, and is part of the Henry et al. (1996) survey. The rotation period is 25 days, like the solar one (Wright et al. 2004). This star will be the top target of nearby stars that closely resemble the Sun for the future Darwin mission (ESA).

HD 146233 (18 Scorpii) has been reported by Porto de Mello \& da Silva (1997) as the best solar twin. Soubiran \& Triaud (2004) confirm this fact in a new search made with the ELODIE library. This is a star of spectral type G2 V and shows an activity cycle at least as long as the Sun, with perhaps a larger amplitude (Hall \& Lockwood 2000). This star is part of the H-K Project and was chosen as a target for the Terrestrial Planet Finder which will search for habitable planets, and for the Darwin project.

HD 19467 is a star of spectral type G3 V with $B-V=0.65$. This star presents low chromospheric Ca II K emission, similar to the Sun.

HD 20766 is an intermediate activity level star, part of the Henry et al. (1996) survey. It is a G2.5 V type star with $B-V=0.64$.

HD 30495 (58 Eridani) is a star of spectral type G3 V, with $B-V=0.64$. This is a star with high a chromospheric activity 
level monitored in the Mount Wilson Observatory, and is part of the Henry et al. (1996) survey. The rotation period is 12 days (Wright et al. 2004).

HD 1835 (BE Cet) has a rotation period of 8 days (Wright et al. 2004). It is a star of spectral type G3 V with $B-V=0.67$, and is part of the H-K Project and of the Henry et al. (1996) survey.

The most active star in the sample is HD 202917. This is a G5 V star with $B-V=0.65$, and is one of the brightest X-ray stars within 50 parsecs of the Sun (Makarov 2003). This star is part of the Henry et al. (1996) survey.

All the stars are part of the library of southern late-type dwarfs published by Cincunegui \& Mauas (2004). The observations were made at the $2.15 \mathrm{~m}$ telescope of the Complejo Astronómico El Leoncito (CASLEO), located in San Juan, Argentina. They were obtained with a REOSC spectrograph designed to work between 3500 and $7500 \AA$ and a $1024 \times$ 1024 pixel TEK CCD as detector. The spectral resolution ranges from 0.141 to $0.249 \AA$ per pixel $(R=\lambda / \delta \lambda \simeq 26400)$. We refer the reader to that work for more details on the observations and the data reduction. For all the stars, we obtained several spectra in different observing runs. In this paper we chose, in all cases, the spectrum with the best signal to noise ratio.

The solar profiles were taken from the FTS atlas (Brault 1999). These observations show the solar absolute diskaveraged flux and were obtained at Kitt Peak Observatory in 1987, when the Sun was in its minimum level of activity.

\section{The modelling}

We built a different model for each star in the sample, assuming a one-dimensional, plane-parallel atmosphere. We solved simultaneously the equations of hydrostatic equilibrium, radiative transfer and statistical equilibrium, using the computer code Pandora. A description of this code can be found in Avrett \& Loeser (2003).

For a given distribution of temperature with height, we selfconsistently computed non-LTE populations for 15 levels of $\mathrm{H}$, 13 of He I, 6 of He II, 15 of Fe I, 8 of Ca I, 5 of Ca II, 7 of Mg I, 6 of Mg II, 21 of Si I, 8 of Na I and 6 of Al I.

From the finished model, we computed the emitted profiles of the Balmer series and of the $\mathrm{Ca}$ II $\mathrm{H}$ and $\mathrm{K}$ line, and modified the model until we found a satisfactory match. The atomic models we used for $\mathrm{H}$ and $\mathrm{Ca}$ II are described in Mauas et al. (1997) and Falchi \& Mauas (1998). The H, K and Ly $\alpha$ lines were computed using Partial Redistribution.

The amount of diagnostic information used to build an atmospheric model is very important. By combining several spectral lines that are formed at different but overlapping depths in the atmosphere, we can obtain a more reliable model. In our case, the spectral features we used to test the models were $\mathrm{H} \beta$ and the $\mathrm{Ca}$ II $\mathrm{H}$ and $\mathrm{K}$ lines. The formation region for the far wings of all these lines is the photosphere. The $\mathrm{K}_{1}$ and $\mathrm{H}_{1}$ absorption minima of the $\mathrm{Ca}$ II lines are formed in the temperature-minimum region. The first chomospheric rise and the beginning of the chromospheric plateau is the region of formation for the $\mathrm{K}_{2}$ and $\mathrm{H}_{2}$ emission maxima of the Ca II lines.

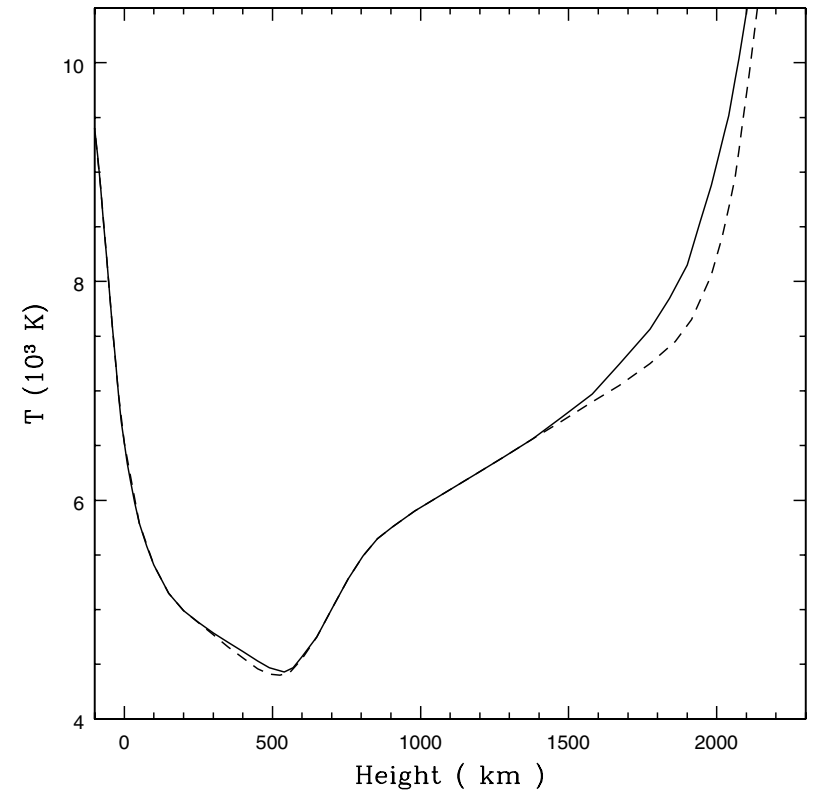

Fig. 1. Our model for the Sun as a star (full line). As a reference, we also show model C by Fontenla et al. (1993) (dashed line).

The absorption cores of the hydrogen and the $\mathrm{Ca}$ II lines $\left(\mathrm{K}_{3}\right.$ and $\mathrm{H}_{3}$ ) are formed from the temperature plateau up to the transition region. We can infer an atmospheric structure by fitting the flux profiles in all these lines.

A successful model should also be able to reproduce line profiles other than those used in its construction. In our case, after the models were finished we checked them comparing the computed profiles of the $\mathrm{Mg} \mathrm{I} \mathrm{b}$ and the $\mathrm{Na}$ I D lines with the observations.

As a first step we built a solar model based on the same spectral features as the stellar models. The most widely used model of the quiet solar chromosphere is model $\mathrm{C}$ by Vernazza et al. (1981), as modified by Fontenla et al. (1993), which was constructed to match the intensities of UV and microwave continua, the Ca II line wing emission, and the hydrogen Ly $\alpha$ line from a mean region on the solar surface, and not to reproduce a specific line profile in detail. In our case, we needed to reproduce the $\mathrm{H} \beta$ and the $\mathrm{Ca}$ II $\mathrm{H}$ and $\mathrm{K}$ line profiles, considering the Sun as a star.

In Fig. 1 we compare our model for the Sun with model C by Fontenla et al. (1993). It can be seen that both models are quite similar. Our model is a little hotter in the temperatureminimum region and in the higher part of the chromospheric plateau, and the transition region is moved inward by $35 \mathrm{~km}$. The profiles we compute from our model are compared with the observations in Fig. 2.

When we constructed the models for the other stars, we tried to reproduce the modelled features with as small a number of changes from the solar model as possible, to avoid overinterpreting the data. To reproduce the asymmetry observed in the Ca II line profiles, in some of the models we included macroscopic velocity fields in the temperature-minimum region and chromosphere.

In Fig. 3 we show the models for all the stars. Concerning the models for the least active stars, including the Sun, the main 


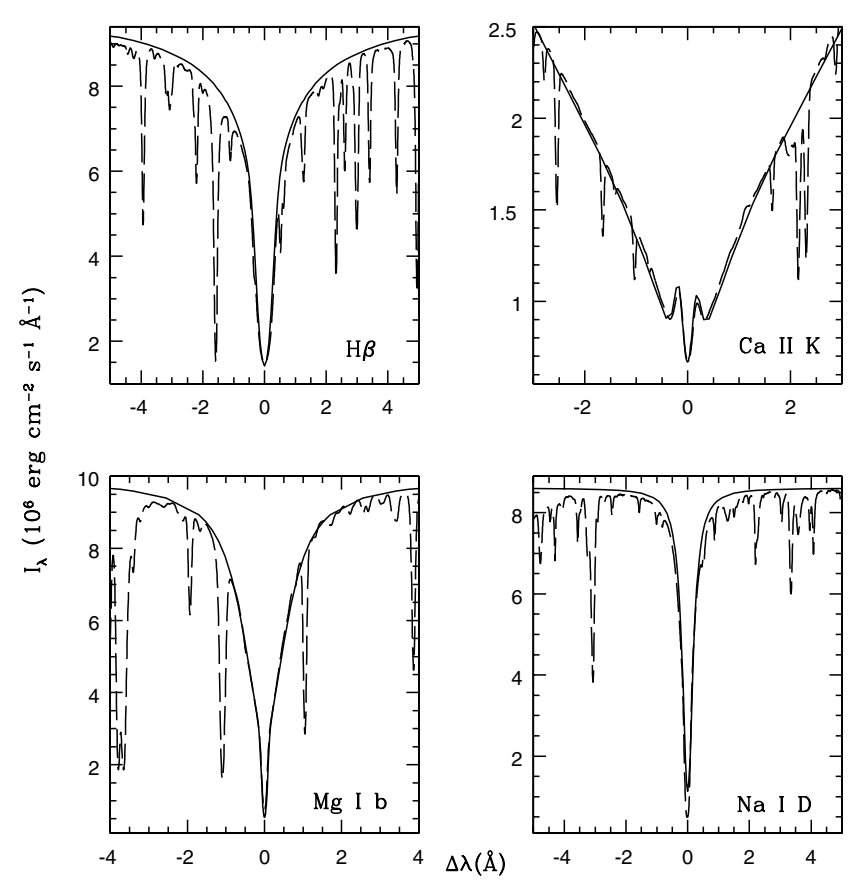

Fig. 2. Observed (dashed line) and synthetic (full line) solar profiles.

change with increasing activity level is in the temperatureminimum region, while the chromospheric plateau and the position of the transition region are the same among all these stars (Fig. 3a).

On the other hand, for the most active stars the entire atmospheric structure is changed, mainly in the upper chromosphere and in the position of the transition region (Fig. 3b).

Regarding the macroscopic velocity fields, which are shown in the upper panels of Fig. 3, we can distinguish between the two groups. The least active group has velocities of the order of $5-10 \mathrm{~km} \mathrm{~s}^{-1}$ only in the chromospheric plateau, while in the most active stars the velocities are of $15-20 \mathrm{~km} \mathrm{~s}^{-1}$, and also the temperature-minimum region is affected by the velocities. Within the most active group the velocity field intensities are independent of the activity level.

The resulting profiles are compared with the observations in Figs. 4 to 12. The observed profiles were converted to the stellar surface using HIPPARCOS parallaxes and the profiles were convolved with a Gaussian function to obtain a profile with the same resolution of the observations. It can be seen that the agreement between the observed and computed profiles is very good.

\section{Energy requirements}

One of the main objectives of this kind of atmospheric modelling is to constrain the amount of chromospheric heating required to sustain the atmosphere of the stars. The total net radiative loss as a function of depth for a given atmospheric model indicates the amount of non-radiative heating necessary to sustain the prescribed temperature structure.
At a given depth the radiative cooling rate $\Phi\left(\mathrm{erg} \mathrm{cm}^{-3} \mathrm{~s}^{-1}\right)$ in a given spectral feature (line or continuum) can be calculated as (Vernazza et al. 1981)

$\Phi=4 \pi \int \kappa_{v}\left(S_{v}-J_{v}\right) \mathrm{d} v$,

where $S_{v}$ is the source function and $J_{v}$ is the mean intensity at frequency $v$. A positive value of $\Phi$ implies a net loss of energy (cooling), and a negative value represents a net energy absorption.

In this section we compute the cooling rate in the lines and continua that are important in cooling and heating the atmosphere. We considered H, H-, H-ff, Mg I and II, Fe I, Si I, Ca II and $\mathrm{CO}$. The total rates for each star are shown in Figs. 13 and 14 for the most and least active stars respectively.

In Figs. 13 and 14 we see that for all the stars the cooling rate is negative in the temperature-minimum region, as was already shown for the Sun (Vernazza et al. 1981). Within the plane-parallel, homogeneous approximation we are investigating, this fact implies either mechanical energy extraction or, more likely, that the calculations have neglected important sources of radiative cooling (see Mauas et al. 1990).

Figure 15 shows the contribution of the most important contributors in this region: $\mathrm{H}-, \mathrm{Si}$ I, Fe I and, particularly, CO, which has been proposed as the main cooling agent at these heights, and as being responsible for a chromospheric bifurcation (e.g. Ayres 1981). However, its contribution is not so large when a detailed calculation is performed (Mauas et al. 1990).

For these calculations we use the Si atomic model studied in Cincunegui \& Mauas (2001), which is much more detailed than the one used in previous papers (Vernazza et al. 1976), and we found a much larger contribution to the cooling in the temperature-minimum region.

However, the Fe I model we used is rather sketchy, and it is possible that a more realistic model of Fe I, taking into account the very large number of Fe I lines that are formed in the upper photosphere and temperature-minimum region, will give a much larger cooling (see Anderson 1989).

Of course, it is also possible that the problem arises from our approximation of a plane-parallel, homogeneous and static atmosphere, very crude in view of the highly dynamic and inhomogeneous atmosphere which is possible to observe in the Sun.

In Fig. 13 it can be seen that the most active stars are closer to radiative equilibrium in this region and the cooling rate is negative in a smaller range, since as the activity level increases the chromospheric heating starts deeper in the first chromospheric rise and is larger throughout the chromosphere.

In Fig. 16 we show, as a function of the Mount Wilson $S_{\text {CaII }}$ index, the cooling-rate integrated in the region where it is positive, from the temperature-minimum to the height in the transition region where the temperature reaches $10^{4} \mathrm{~K}$. The integrated cooling rate represents the total mechanical energy necessary to sustain the atmosphere of the star. In Fig. 16 we also included model P of Fontenla et al. (1993) and model FLA of Mauas et al. (1990), representing a solar plage and a solar flare respectively. 

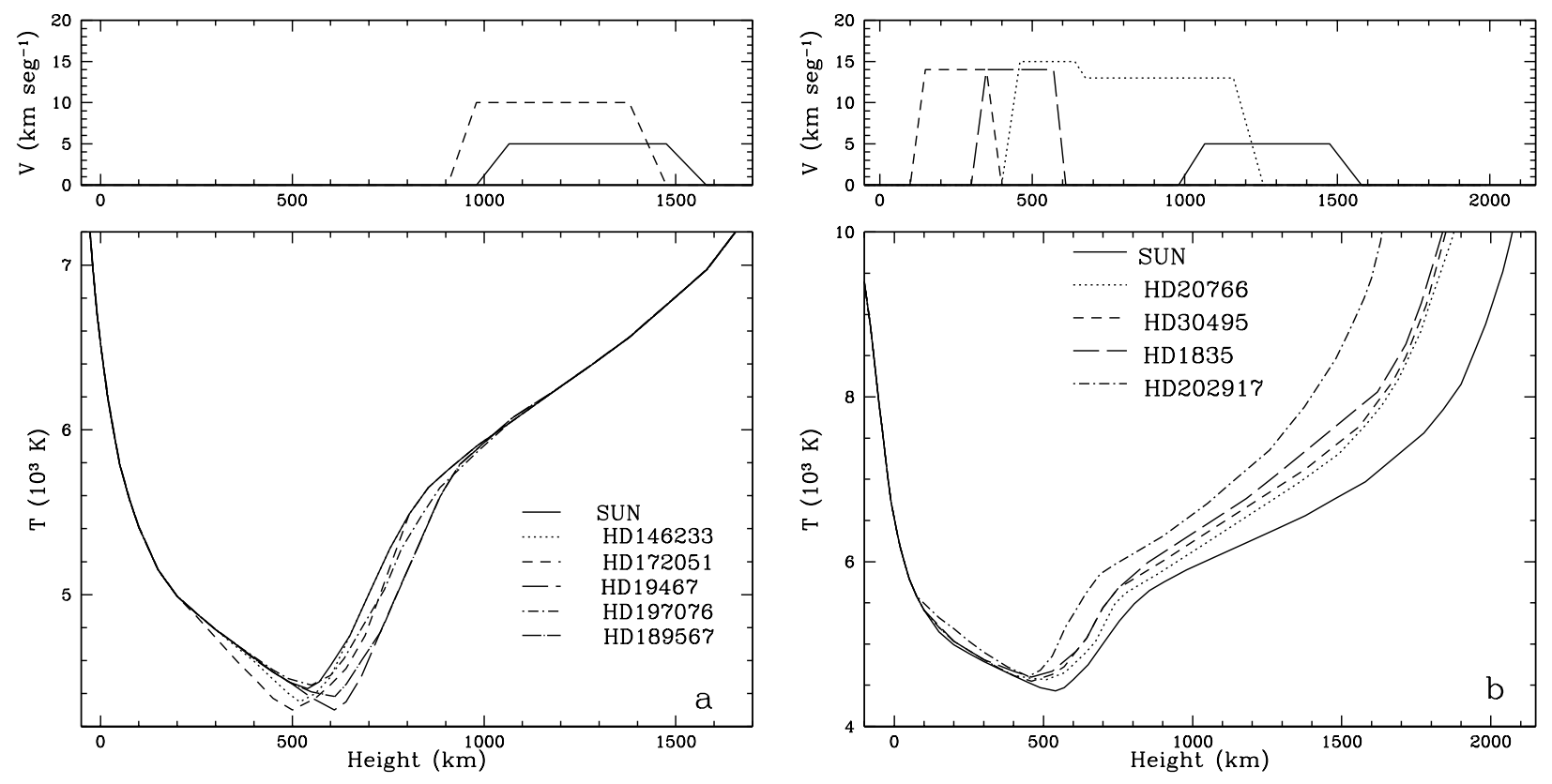

Fig. 3. a) Models of the least active stars. b) Models of the most active stars. Top: macroscopic velocity fields for the corresponding star.

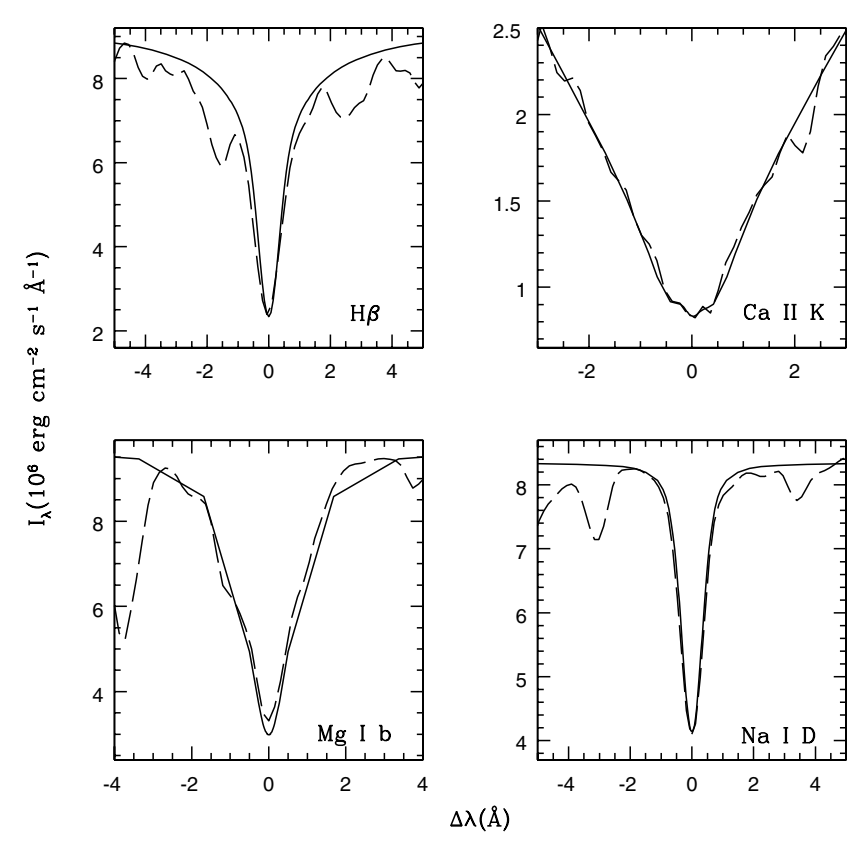

Fig. 4. Observed (dashed line) and computed (full line) profiles for HD 146233.

In all cases, the values of $S_{\text {CaII }}$ shown in Fig. 16 were measured in the computed profiles, obtained from the models as were the $\Phi_{\text {int }}$. In this way, we avoid propagating the differences that may occur between observed and computed profiles. In particular, many of these stars show strong variability, and the models we obtained represent snapshots of the state of the stars at a given moment. In a different moment, a star may be more or less active, and a different model would be appropriate, from which we would obtain larger or smaller values of $S_{\text {CaII }}$ but also larger or smaller energy requirements, as given by $\Phi_{\text {int }}$.

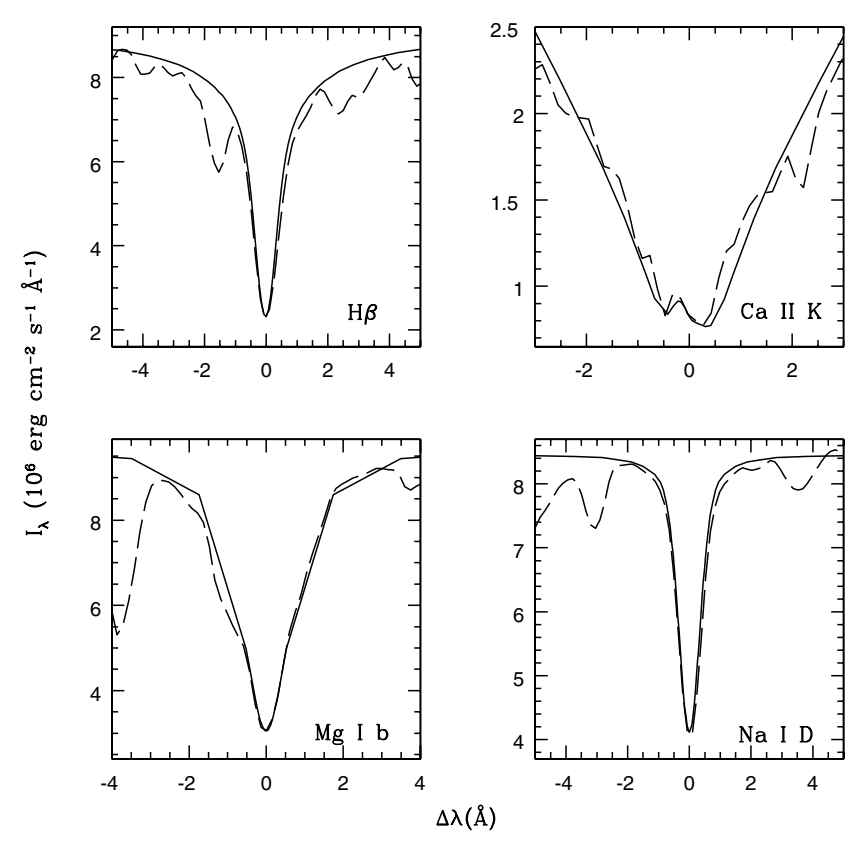

Fig. 5. Observed (dashed line) and computed (full line) profiles for HD 172051.

It can be seen that there is a very good linear regression between these two quantities (represented by the line in the figure), which is given by

$\Phi_{\text {int }}=4507 * S_{\text {CaII }}-678$

and has a correlation coefficient $r=0.989$. This implies that $S_{\text {CaII }}$ is in fact a good proxy of stellar activity, giving a direct measure of the quantity of energy necessary to sustain the atmosphere.

We note that Eq. (2) implies a minimum value for $S_{\text {CaII }}$ of 0.15 , which would correspond to an atmosphere in radiative equilibrium $\left(\Phi_{\text {int }}=0\right)$. However, as it is clear from the figure, 


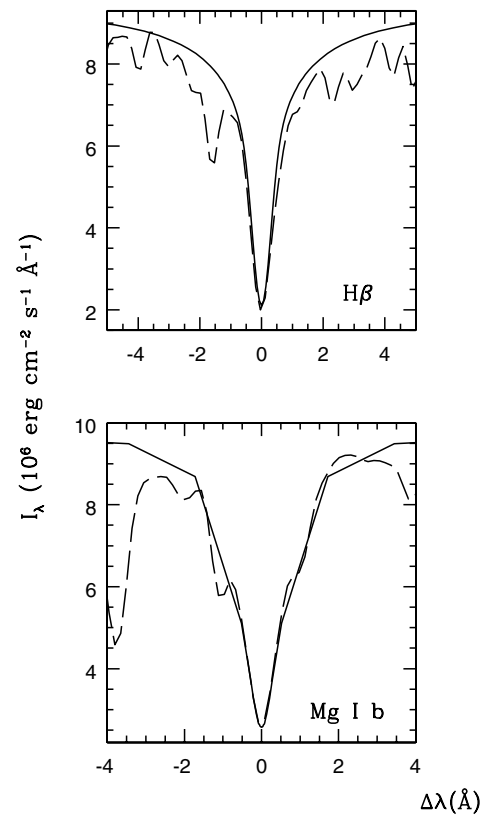

Fig. 6. Observed (dashed line) and computed (full line) profiles for HD 19467.

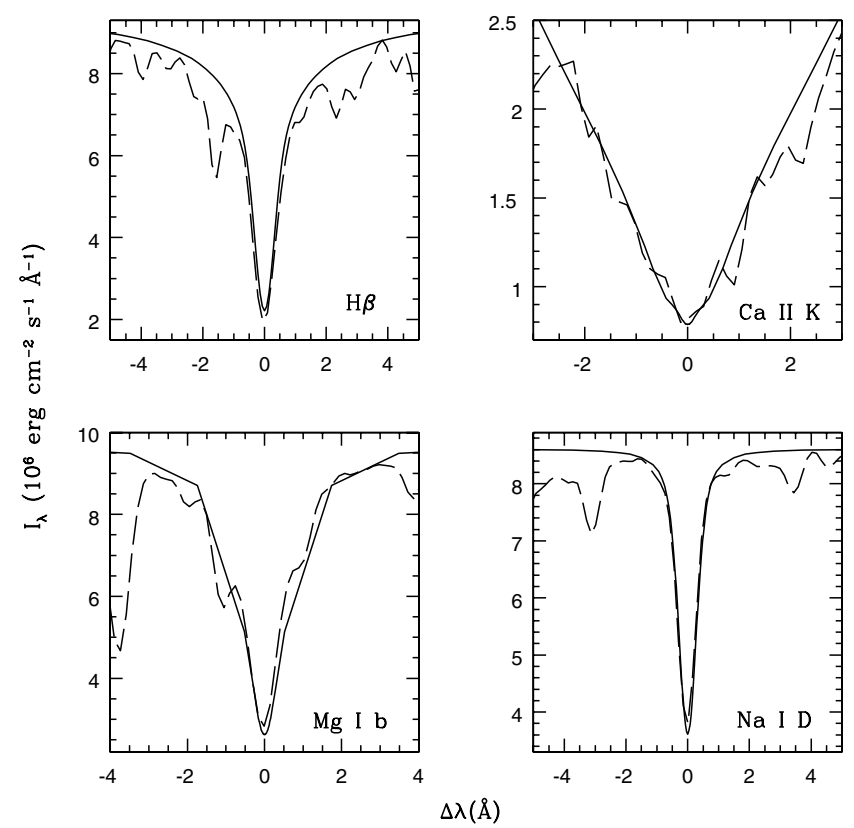

Fig. 7. Observed (dashed line) and computed (full line) profiles for HD 197076.

the fit depends very heavily on the two points at $S_{\text {CaII }} \simeq 0.6$. If we compute the regression without considering these two points, we obtain $\Phi_{\text {int }}=3448 * S_{\text {CaII }}-458$, which implies a smaller value for the minimum $S_{\text {CaII }}$ of 0.133 .

On the other hand, closer to radiative equilibrium smaller contributions to the cooling rate could become important, in particular the effect of the possible cooling agents missing in our calculations, as discussed in Sect. 4. Nevertheless, these values should serve as an indication of the minimum value of $S_{\text {CaII }}$ for a star of the same characteristics of the Sun. This,
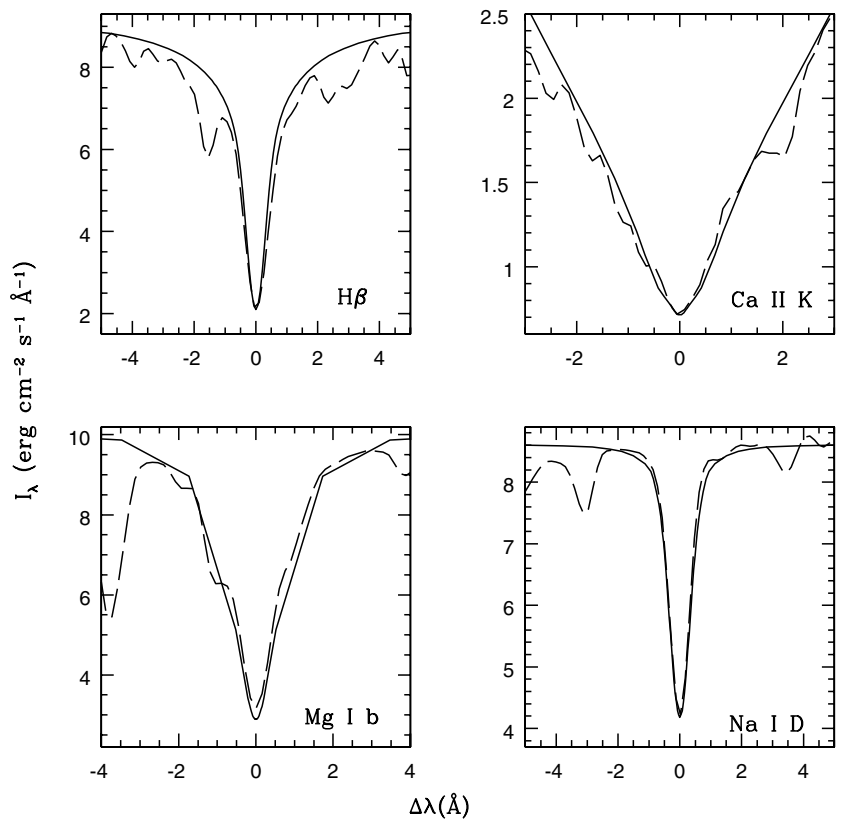

Fig. 8. Observed (dashed line) and computed (full line) profiles for HD 189567.

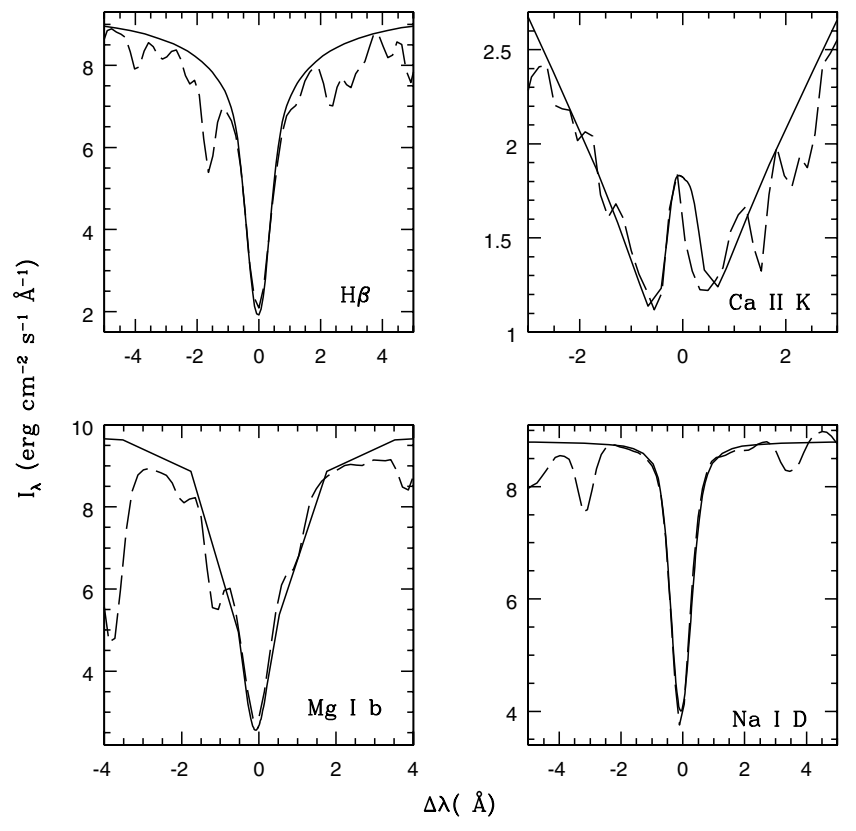

Fig. 9. Observed (dashed line) and computed (full line) profiles for HD 20766.

in particular, can be relevant for the reconstruction of the solar irradiance during periods of very small levels of solar activity like the Maunder Minimum. We note that the value for $S_{\text {CaII }}$ estimated for the Sun in the Maunder Minimum by Henry et al. (1996) is 0.145 , close to our minimum value for this index.

\section{Discussion}

In this paper we present chromospheric models for the Sun as a star and for nine solar analogues, which show a wide variety of activity levels. 


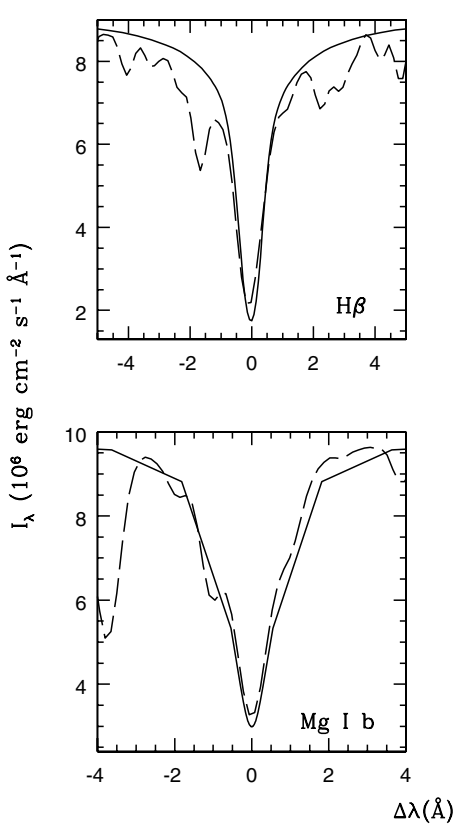

Fig. 10. Observed (dashed line) and computed (full line) profiles for HD 30495.

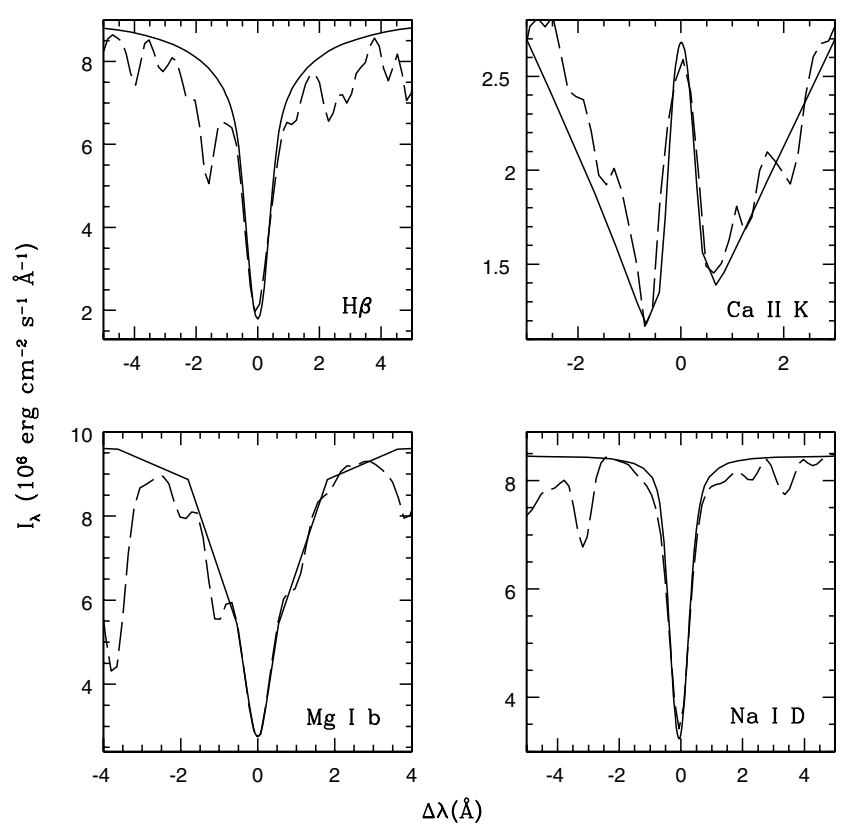

Fig. 11. Observed (dashed line) and computed (full line) profiles for HD 1835.

These models were based on, and reproduced very well, the $\mathrm{Ca}$ II $\mathrm{H}$ and $\mathrm{K}$ and the $\mathrm{H} \beta$ line profiles. The reliability of the stellar atmospheric models was checked with other features, the $\mathrm{Na} I \mathrm{D}$ and $\mathrm{Mg}$ I $\mathrm{b}$ lines. Also for these lines we found very good agreement between computed and observed profiles.

All the G-type stars in our sample show an atmospheric structure similar to the solar one. Each star presents a temperature-minimum region, a chromospheric rise followed by a plateau, and a steep temperature rise to the transition region. These confirm and expand the results of Franchini et al. (1998) and Morossi et al. (2003) who using a different
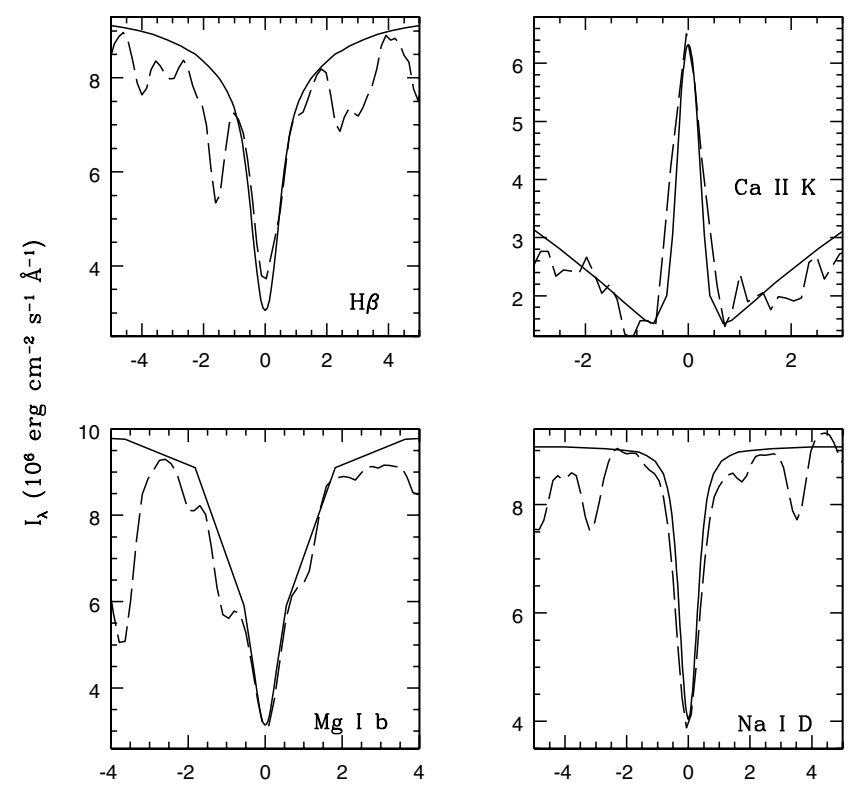

Fig. 12. Observed (dashed line) and computed (full line) profiles for HD 202917.

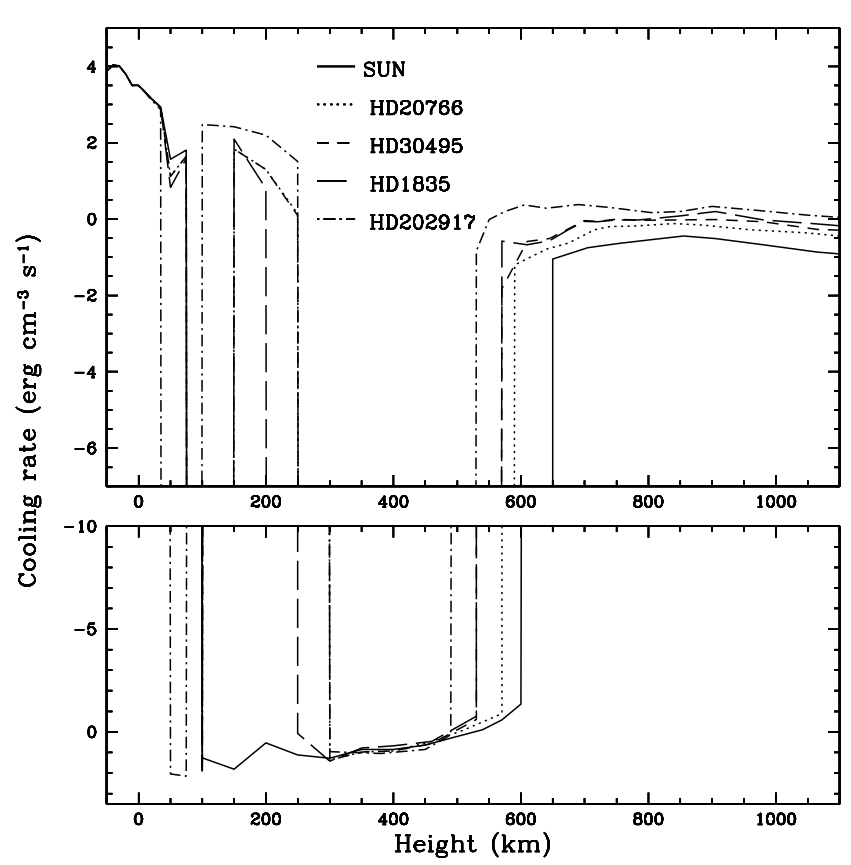

Fig. 13. Total cooling rate of the most active stars.

wavelength range, the ultraviolet continua, also found that a chromospheric temperature rise was necessary.

Pasquini (1992) studied observations of the Ca II K line for a sample of solar-type stars (G0-G5 in spectral type) and found that, as activity increases, first the intensity of the $\mathrm{K}_{1}$ minimum increases, and for larger activity levels also the intensity of the $\mathrm{K}_{2}$ maxima and of the central reversal $\mathrm{K}_{3}$ change. This result is well reproduced by the changes in the atmospheric structure along the stellar sample discussed in Sect. 3, where we found that the first changes with increasing activity are in 


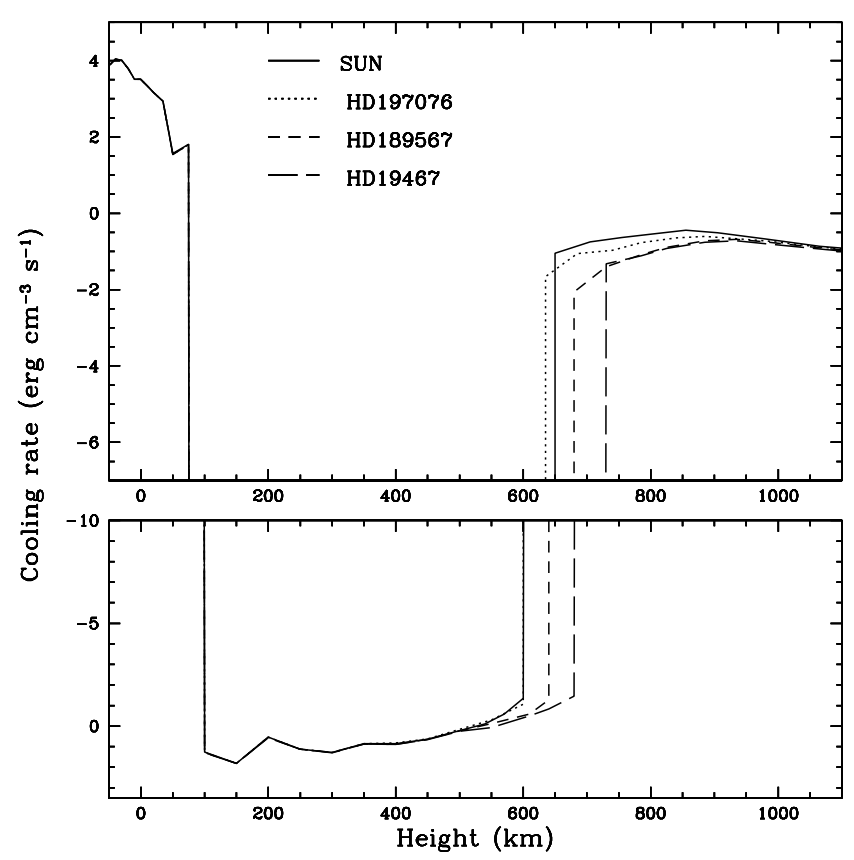

Fig. 14. Total cooling rate for some of the least active stars.

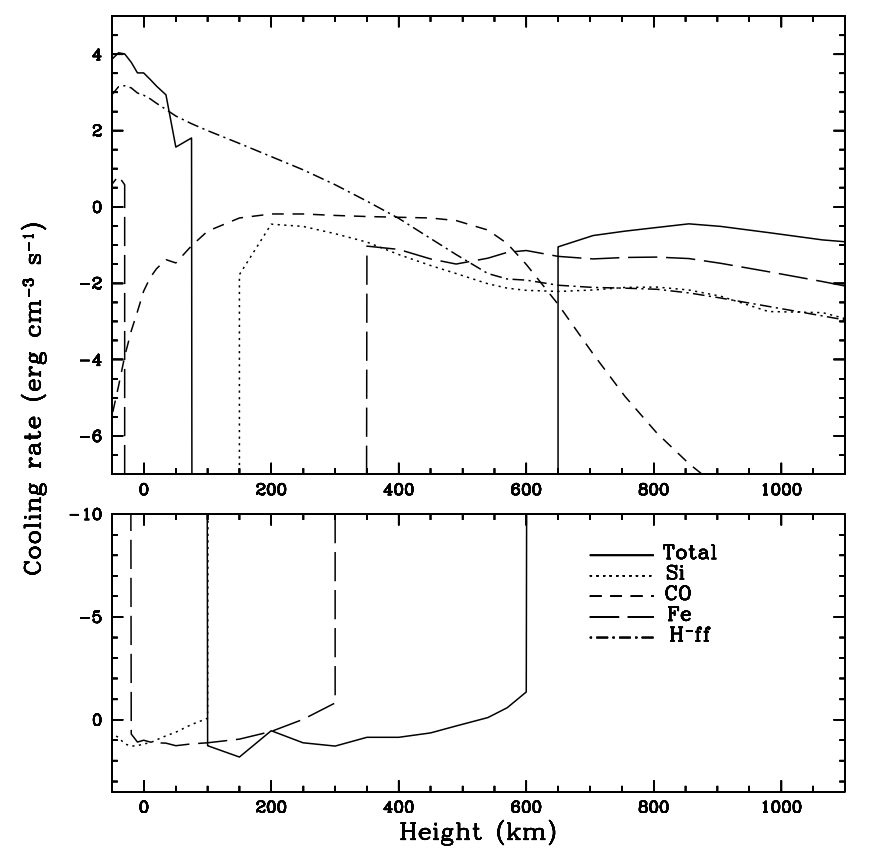

Fig. 15. Cooling rate in the temperature-minimum region for the Sun, and its main components.

the temperature-minimum region and afterwards in the chromospheric plateau and in the higher chromosphere.

On the other hand, the response of the Ca II line profiles to activity is not linear with changes in the atmospheric structure, as can be seen comparing the models and the corresponding Ca II line profiles. As the stellar activity level increases the line profiles become more sensitive to changes in the temperature of the atmosphere, due to the increasing role of collisional processes. This effect was discussed by Vieytes \& Mauas (2004),

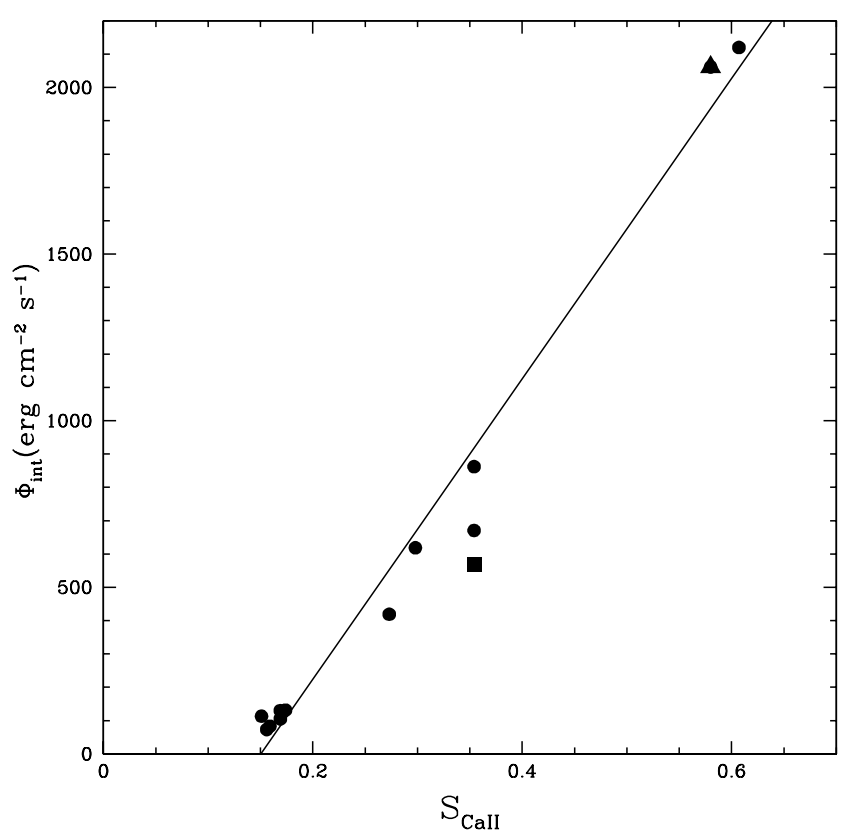

Fig. 16. Integrated cooling-rate as a function of the $S_{\text {CaII }}$ index. The line is the fit to the data given by (2), model P of Fontenla et al. (1993, square), and model FLA of Mauas et al. (1990, triangle).

who found that it can explain the so-called Vaughan-Preston gap.

Concerning the energetic requirements, all the stars show a negative cooling rate in a region around the temperatureminimum. As the stellar activity increases, this region is smaller and the chromospheric heating starts deeper in the first chromospheric rise. We also found a very good linear relation between the integrated cooling rate and the activity index $S_{\text {CaII }}$, which can therefore be used to estimate the star's energy requirements from its activity level.

Acknowledgements. We would like to thank the director of the CASLEO Observatory, Dr. H. Levato, and all the staff of this institution. This work made extensive use of the SIMBAD database, operated at CDS, Strasbourg, France.

\section{References}

Anderson, L. S. 1989, ApJ, 339, 558

Avrett, E., \& Loeser, R. 2003, in Modeling of Stellar Atmospheres, IAU Symp., 210, ed. W. Weiss, \& N. Piskunov (Dordrecht: Kluwer), A-21

Ayres, T. R. 1981, ApJ, 244, 1064

Baliunas, S., Donahue, R. A., Soon, W. H., et al. 1995, ApJ, 438, 269

Baliunas, S., Donahue, R., Soon, W. H., \& Henry, G. 1998, Cool Stars, Stellar Systems and the Sun (ASP), 154, 153

Brault, J., \& Neckel, H. 1999, Sol. Phys., 184, 421

Carlsson, M., \& Stein, R. 1997, ApJ, 481, 500

Cincunegui, C., \& Mauas, P. 2001, ApJ, 552, 877

Cincunegui, C., \& Mauas, P. 2004, A\&A, 414, 699

Duncan, D., Vaughan, A., Wilson, O., et al. 1991, ApJS, 76, 383

Falchi, A., \& Mauas, P. J. D. 1996, A\&A, 310, 245

Falchi, A., \& Mauas, P. J. D. 1998, A\&A, 336, 281

Fontenla, J., Avrett, E., \& Loeser, R. 1993, ApJ, 406, 319

Franchini, M., Morossi, C., \& Malagnini, M. 1998, ApJ, 508, 370 
Hall, J., \& Lockwood, G. 2000, ApJ, 545, L43

Henry, T., Soderblom, D., Donahue, R., \& Baliunas, S. 1996, AJ, 111, 439

Houdebine, E., \& Doyle, J. 1994a, A\&A, 289, 169

Houdebine, E., \& Doyle, J. 1994b, A\&A, 289, 185

Houdebine, E., Doyle, J., \& Kościelecki, M. 1995, A\&A, 294, 773

Houdebine, E., Mathioudakis, M., Doyle, J., \& Foing, B. 1996, A\&A, 305, 209

Makarov, V. 2003, AJ, 126, 1996

Mauas, P. J. D., Avrett, E. H., \& Loeser, R. 1990, 357, 279

Mauas, P. J. D., \& Falchi, A. 1994, A\&A, 281, 129

Mauas, P. J. D., Falchi, A., Pasquini, L., \& Pallavicini, R. 1997, A\&A, 326,256
Mauas, P. J. D., Machado, M. E., \& Avrett, E. H. 1990, ApJ, 360, 715

Morossi, C., Franchini, M., Malagnini, M., \& Chavez, M. 2003, in the 12th Cambridge Workshop on Cool Stars, Stellar Systems and the Sun, 285

Neckel, H. 1986, A\&A, 159, 175

Pasquini, L. 1992, A\&A, 266, 347

Porto de Mello, G., \& Da Silva, L. 1997, ApJ, 482, L89

Soubiran, C., \& Triaud, A. 2004, A\&A, 418, 1089

Thatcher, J., Robinson, R., \& Rees, D. 1991, MNRAS, 250, 14

Vernazza, J., Avrett, E., \& Loeser, R. 1976, ApJS, 30, 1

Vernazza, J., Avrett, E., \& Loeser, R. 1981, ApJS, 45, 635

Vieytes, M., \& Mauas, P. 2004, Ap\&SS, 290, 311

Wright, J., Marcy, G., Butler, R., \& Vogt, S. 2004, ApJS, 152, 261 